\title{
THE MATHEMATICAL APPROACH TO THE SONIC BARRIER ${ }^{1}$
}

\author{
BY CATHLEEN SYNGE MORAWETZ
}

1. Introduction. For my topic today I have chosen a subject connecting mathematics and aeronautical engineering. The histories of these two subjects are close. It might however appear to the layman that, back in the time of the first powered flight in 1903, aeronautical engineering had little to do with mathematics. The Wright brothers, despite the fact that they had no university education were well read and learned their art using wind tunnels but it is unlikely that they knew that airfoil theory was connected to the Riemann conformal mapping theorem. But it was also the time of Joukowski and later Prandtl who developed and understood that connection and put mathematics solidly behind the new engineering. Since that time each new generation has discovered new problems that are at the forefront of both fields. One such problem is flight near the speed of sound. This one in fact has puzzled more than one generation.

Everyone knows that in popular parlance an aircraft has "crashed the sonic ". or sound barrier" if it flies faster than sound travels; that is, if the speed of the craft $q$ exceeds the speed of sound $c$ or the Mach number, $M=q / c>1$. In simple, if perhaps too graphic, terms this means that if you are in the line of flight the plane hits you before you hear it. But it is not my purpose to talk about flight at the speed of sound. The mathematical and engineering problems today are centered around a different range called the transonic range where $M$ is roughly between 0.6 and 0.9 . We shall see that this is where the real sound barrier lies. But the main reason for working in this range is that we can expect, because of the energy shortage, to fly for a good many years at those speeds. We need to understand them and find the best way to do it. In the first part of my talk I want to explain where the energy benefits come from and something of the history of this type of flight. After that, I shall turn to the mathematics.

People have always dreamed of flying. In ancient times-like the birds. In modern times like Buck Rogers. For a long time we have known that if you have a big enough engine, creating lots of thrust, you can fly anywhere and theoretically at any speed. Like Buck Rogers you do not need wings. It costs money but it can be done. But back in the Wright brothers' day there was no

The Josiah Willard Gibbs lecture was presented at the 783rd meeting of the American Mathematical Society in San Francisco, California, January 7, 1981; received by the editors July $15,1981$.

1980 Mathematics Subject Classification. Primary 76H05; Secondary 35M05.

1 The author gratefully acknowledges support over many years by the Office of Naval Research and currently from Grant No. N00014-76-0439. 
way to use what energy there was, everything was too heavy, engines were weak and to fly at all one had to have a good pair of wings, i.e., a well made airfoil or wing cross-section.

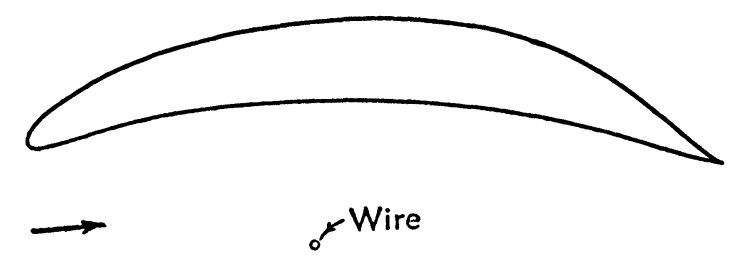

FIGURE 1. Airfoils of equal drag experimentally

An illuminating comparison is to be found in R. T. Jones' book [22], see Figure 1. For incompressible flow, which for us means low speed with a given thrust, or drag, which is equivalent to energy, you can fly either the little wire cylinder or the big air foil obtained by E. Jacobs by a conformal map; obviously the big one is better. More room for the gas tanks, for cargo, etc. But more importantly the shape gives lift. What is at work? The principle of flying is the same as the principle of sailing into the wind. The high school physics explanation is that the plane is sucked upwards or the sail is sucked forward by a partial vacuum created by the very special shape of the airfoil of sail. That is basically right. Now sailing saves a lot of energy even if you include the energy of the wind. So it is not unreasonable to believe that we can save energy by flying in, what I like to call, the "sailing mode". That is, getting as much as possible of the free ride the sailor has by designing the wings to help in the motion. But how far can we push this idea? It turns out there are limitations. The faster we fly the more Buck Rogerish we become and trouble comes at Mach numbers less than 1.

In Figure 2, we have a look at this with some airfoils in wind tunnels. These are Schlieren photos of an airfoil beginning in (a) at low speed. There is a wind blowing on a fixed airfoil so now the Mach number is that of the wind. In a Schlieren photograph gradients of pressure show up as shadows. So for example we see the trail of vortices that provides the lift that keeps the airfoil up as well as the drag which has to be compensated for by thrust. One also sees the viscous boundary layer, separating a little near the trailing edge. That must be controlled to avoid stalling and kept down and the trick is to have a sizable pressure gradient at the nose.

We are going to have to distinguish between the overall Mach number, denoted by $M_{\infty}$ which is the ratio of the wind speed to the speed of sound at $\infty$ and the local Mach number, $M$, the ratio of the two speeds at a particular point. As $M_{\infty}$ increases the flow becomes locally supersonic, the local Mach number exceeds 1 in a bubble adjacent to the airfoil, a "supersonic bubble". This is when $M_{\infty}$ enters the transonic range, as in (b). Two things are happening: noise is propagated locally so the flow is not very smooth and a shock, a discontinuity in pressure, appears. Figure $2 \mathrm{c}$ is of a different airfoil in the same stage as $2 b$; the single shock happens to be sharper. 

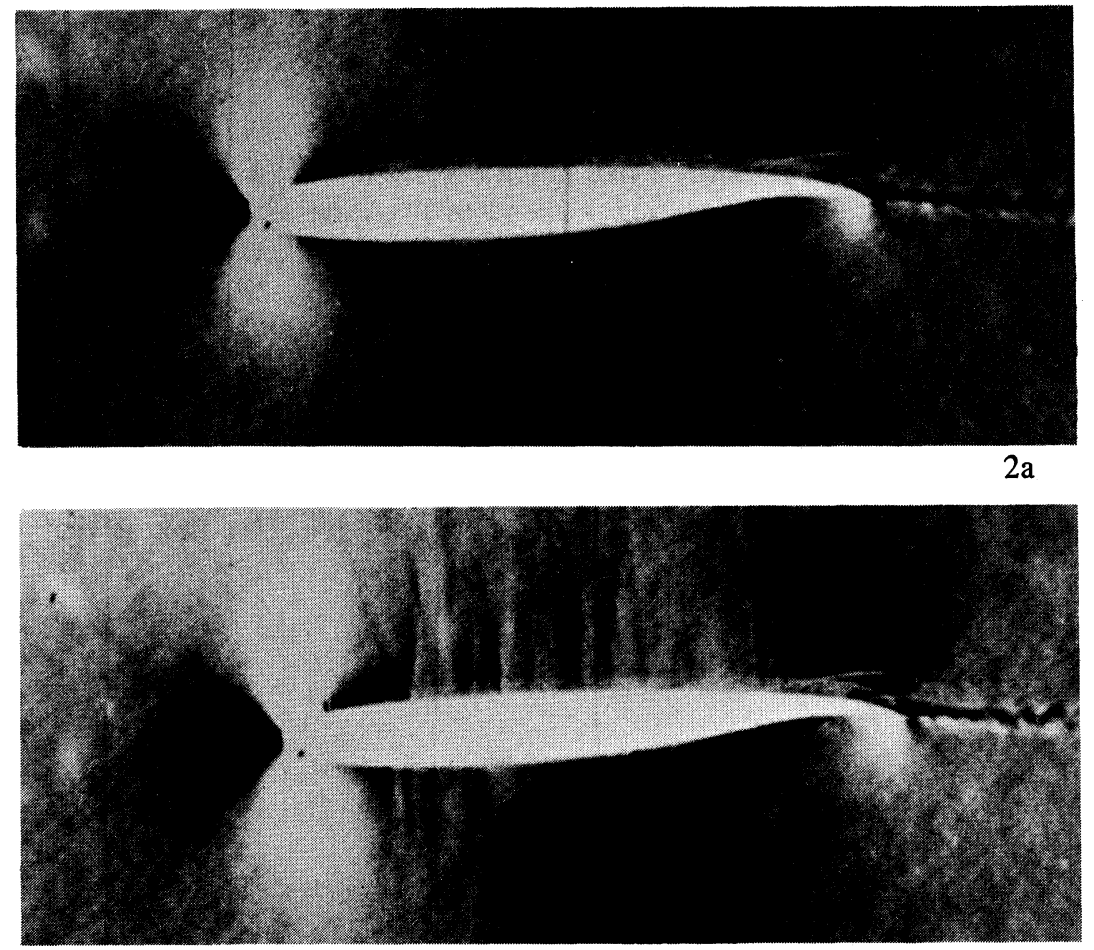

$2 b$
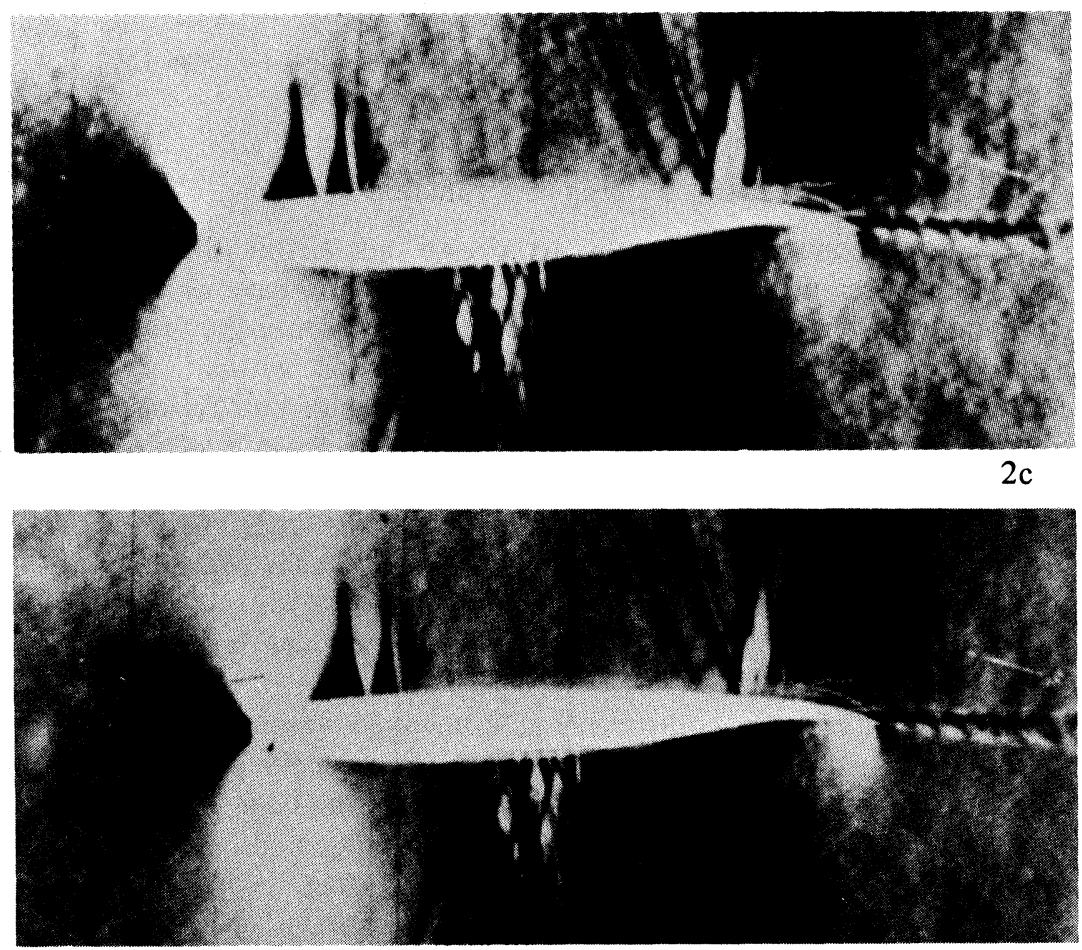

FIgURE 2. Wind tunnel experiments 
As the speed is increased still further a second shock on the lower side develops, Figure 2d. Eventually the two shocks move backwards and sit off the trailing edge in what is called a fish tail configuration. And, finally, of course, $M_{\infty}$ exceeds 1 and a shock appears ahead of the wing as with the Concorde plane and we enter another regime, from the point of view of energy consumption.

As we proceed through these stages what happens to the energy requirements? Energy behaves like thrust or drag. We normalize it and make it dimensionless essentially by the square of the speed.

The drag, mainly due to viscous effects, is going up like the square of the velocity, see Figure 3, the heavy curve, and then it hits a dramatic rise which is due to the presence of shocks in the supersonic bubble. This then is the true sonic barrier.

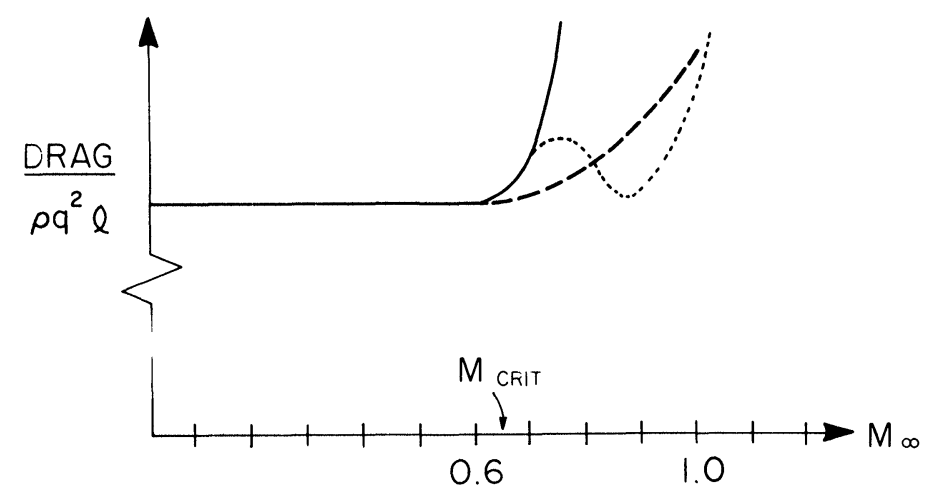

Figure 3. Drag as a function of $M_{\infty}$

The aerodynamical problem is to design an airfoil that will fit all aerodynamical requirements. It should be of good size, have no separation of the boundary layer and at the same time should minimize the drag. Since the shocks cause the drag why not look for an airfoil without shocks? This question was first raised about 1930 when G. I. Taylor and Busemann began exploring the problem, Taylor [42] developing some smooth locally transonic flows and Busemann [5] getting contradictory wind tunnel results. This was not actually the beginning. In one form or another the question goes back to Chaplygin [7], Lord Rayleigh [39] and in more detail to Th. Meyer [30] in 1908.

In the following thirty to forty years there was time for a large measure of controversy followed by indifference. Nowadays we are back with a small measure of controversy.

The ingredients for the controversy as it emerged were twofold. You could apparently construct a family of airfoils depending on the parameter $M_{\infty}$. To each $M_{\infty}$, over some range, there was one airfoil that had one smooth flow with a supersonic bubble. At any other value of $M_{\infty}$ nothing could be said.

Thus for one choice of $M_{\infty}$ there was no extra drag. For any other-who knew? Still it was an indication that one might build airfoils with no shocks, no 
gratuitous drag. But the available computers were not good enough to actually produce one of these airfoil designs.

On the other side, wind tunnels reported shocks under all circumstances as soon as the supersonic bubble appeared. But the wind tunnel experiments were very difficult and perhaps they were not properly interpreted.

The controversy boiled down to the question: how exceptional were the special airfoils?

Eventually the matter was settled (there are still diehards on the other side) by a good dose of mathematics first proposed by engineers; the special airfoils can be found; they are exceptional. But what about the next question? What really happens to these airfoils at what we call off-design conditions? But by this time we were into the fifties. Sputnik had risen and the indifference to transonic flow set in. All hands turned to getting a man on the moon. That put the "sailing" flight program on the back burner. Buck Rogers was in. We were going to go everywhere by rocket. Limits were imposed on new wind tunnels. Old ones were dismantled. One cannot do everything. And the obvious spin-off was going to be very fast earth-to-earth flight. Who cares about a few meager points in the Mach number? Then came the seventies and the energy crunch. The indifference ended. Buck Rogers was out. "Sailing" flight was in. Those special airfoils could then be found with the new computers and tested in new wind tunnels. In our drag graph, Figure 3, they give a dip (...) at some $M_{\infty}$. In actual practice one aims only close to one of these special designs; the drag curve is smeared out (---). The new airfoils have shocks but they are weak. Their drag is much lower than say that of a Joukowski airfoil. A few meager points of Mach number are very important. They are worth a lot of oil and a lot of dollars. So the upcoming generation of airplanes, the many small jets already in action are all flying transonically. In the future when you look out of the window of your jet plane you may even see little shock waves sitting on its surface but you will know that the wing has been designed to keep them harmless.

2. The mathematical model. So what is the mathematics that does all this for us?

First, the mathematical model. Of course, air is viscous as well as compressible but except for boundary layers it has proved up to now that a very satisfactory model for flight is given by treating the flow as compressible, irrotational and of constant entropy.

The variables are then density $\rho$, velocity $\vec{q}$; pressure is a function of $\rho$. We consider the airfoil at rest so that the velocity at $\infty, \vec{q}_{\infty}$, is really the velocity of the plane. From irrotationality there exists a potential so that $\vec{q}=\nabla \phi$ and from the conservation of momentum one finds Bernoulli's law (its incompressible form was used in those high school "vacuum" arguments) and yields a relation between $p, \rho,|\nabla \phi|$. We may take it in the form

$$
\rho=\rho(|\nabla \phi|) \text {. }
$$

As the remaining physical law we have, conservation of mass:

$$
\operatorname{div}(\rho \nabla \phi)=0 \text {. }
$$


This nonlinear second order differential equation replaces the harmonic equation $\Delta \phi=0$ of incompressible flow.

These equations can be written in a particularly elegant form, if there are only two space variables, $d \phi+\rho^{-1} d \psi=w d z$ analogous to $d \phi+i d \psi=w d z$ where $\vec{q}=(u, v)$ has been replaced by the complex velocity, $w=u-i v$ and $z=x+i y$, and $\psi$, the stream function, is defined through this form.

The elegance however conceals the very special feature of the system which shows up if we rewrite the equation for $\phi$,

$$
\left(c^{2}-u^{2}\right) \phi_{x x}-2 u v \phi_{x y}+\left(c^{2}-v^{2}\right) \phi_{y y}=0
$$

where $c=(d p / d \rho)^{1 / 2}=c(|\nabla \phi|)$ is the speed of sound, given through Bernoulli's law. For example, for air $c^{2}=.2\left(q_{*}^{2}-q^{2}\right)$ where $q_{*}$ is a constant. If $u, v$ are small we have $\Delta \phi=0$; the flow is essentially incompressible. Choosing coordinates locally so that $v=0$ we see that, for $q<c$ the equation is elliptic and for $q>c$ the equation is hyperbolic. So for $q<c$ the flow is completely analogous to the incompressible case, its solutions are locally smooth, but for $q>c$, all the difficult features of nonlinear hyperbolic equations occur.

Equation (1) is to hold outside the airfoil, in $\mathscr{D}$; in addition, the normal component of velocity must be zero on the airfoil

$$
\partial \phi / \partial n=0 \text { on } \partial \mathscr{D}
$$

or equivalently $\psi=$ constant and at $\infty$, the flow attains a prescribed velocity

$$
\nabla \phi \rightarrow \nabla \phi_{\infty}, \quad|x| \rightarrow \infty
$$

That turns out to be sufficient data provided we are thinking of wings with sharp trailing edges. In the incompressible case we force the solution to have finite velocity there (the Kutta-Joukowski condition). In the compressible case infinite velocities are impossible anyway. For air, $c^{2}=0$ for $q=q_{*}$ and thus a vacuum occurs instead of infinite speed. In the compressible case what we want is the right pattern for the streamlines. Thus we require

The Kutta-Joukowski condition: The streamlines ( $\psi=$ const) along the airfoil join at the trailing edge and exactly one streamline issues from the trailing edge.

This form of the Kutta-Joukowski condition seems to hold even in the extreme case of the fishtail shock of Figure 2e.

We are looking at the boundary value problem given by (1)-(4) but we must consider what class of functions should describe the solution. One's first hope is for $C^{\infty}$ solutions if the airfoil is $C^{\infty}$ except at the trailing edge. But the experiment has suggested that for transonic flows this will be impossible. So the problem of existence and uniqueness is first treated for values of $M_{\infty}$ below $M_{\text {crit }}$ when the equations are elliptic. Of course one has to establish that there exists an $M_{\text {crit }}>0$. All the elegant methods of nonlinear elliptic equations can be used. In fact the elliptic theory and the subsonic flow theory came together. During the fifties, Schiffman [41], Bers [3] established the general existence and uniqueness (as subsonic flows) using a variety of methods, see also [17]. 
Recently, Brezis, Stampacchia [4], Hummel [20] have considered the problem for convex bodies by a new variational approach.

What about Mach numbers higher than $M_{\text {crit }}$ ? No existence or uniqueness theorems exist. How can the problem be approached? Can we continue to work with smooth solutions? The wind tunnel experiments tell us certainly not always.

3. Constructing a smooth transonic flow. Since we explicitly want the exceptional cases of smooth flow we begin by showing how to construct an airfoil numerically that does have a smooth flow and a supersonic bubble.

We could begin in the following way. Suppose on a very large circle we prescribe Cauchy data $(\phi, \partial \phi / \partial n)$ that is real analytic. This data can be extended analytically as a solution of our nonlinear equation by the CauchyKowalewski theorem.

Most such solutions will develop a singularity. But some may produce a streamline $(\psi=$ constant $)$ that divides and then closes and it does not matter if there are singularities only inside. If the speeds are high enough it might have a supersonic bubble. Thus we would have the desired flow past a profile. If we can push our circle "to $\infty$ " we would get a flow in the whole domain exterior to the profile.

For an incompressible flow, the complex velocity $w$ is analytic and, since it approaches a constant at $\infty$, it has a power series expansion in $z^{-1}$. Pushing our circle to $\infty$ means prescribing all of the terms of this expansion. There is a similar rather messy series for $w$ as a function of $x, y$ for the compressible case. It happens that the corresponding potential $\phi$ has a unique continuation in the $x, y$ plane.

This idea of using such a series goes back to Chaplygin [7], Cherry [8] and Lighthill $^{2}$ [28], who showed how to construct solutions for $\phi$, actually in the velocity plane $(u, v)$ where the equations become linear and can be separated. Nieuwland [36] constructed the first effective computational examples in 1964. It is amazing, considering how slow machines were and how complicated the series were, that he could get enough significant figures to show he really had an airfoil (roughly elliptic in shape).

Garabedian's approach to this problem [2], which produces useful airfoils extremely efficiently, has three main ingredients:

(i) Extend the two-dimensional real space to complex space and then treat the equations hyperbolically,

(ii) replace the analytic continuation process by a difference scheme,

(iii) prescribe data so that an airfoil with a supersonic bubble is produced.

For (i) the variables $x, y, \phi, u, v$, become complex. The physical plane is $\operatorname{Im} x=\operatorname{Im} y=0$. Treating the equations hyperbolically means using characteristics.

Let us look at the simple case of the Cauchy-Riemann equations, which govern the incompressible case,

$$
u_{x}+v_{y}=0, \quad u_{y}-v_{x}=0
$$

\footnotetext{
${ }^{2}$ See the bibliography in the book by Bers for other approaches.
} 
They can be rewritten, if we set $x=X, u=U, y=i Y, v=-i V$, as

$$
U_{X}-V_{Y}=0, \quad V_{X}-U_{Y}=0,
$$

a familiar hyperbolic system.

In characteristic form for the four variables $X, Y, U, V$, we have

$$
\begin{array}{ll}
Y_{\xi}+X_{\xi}=0, & U_{\xi}+V_{\xi}=0, \\
Y_{\eta}-X_{\eta}=0, & U_{\eta}-V_{\eta}=0,
\end{array}
$$

or

$$
\begin{array}{ll}
y_{\xi}+i x_{\xi}=0, & u_{\xi}+i v_{\xi}=0, \\
y_{\eta}-i x_{\eta}=0, & u_{\eta}-i v_{\eta}=0 .
\end{array}
$$

If we do the same for the compressible case, we find

$$
\begin{array}{ll}
y_{\xi}+\lambda_{+} x_{\xi}=0, & u_{\xi}-\lambda_{-} v_{\xi}=0, \\
y_{\eta}+\lambda_{-} x_{\eta}=0, & u_{\eta}-\lambda_{+} v_{\eta}=0,
\end{array}
$$

with

$$
\lambda_{ \pm}=\frac{u v \pm c \sqrt{q^{2}-c^{2}}}{c^{2}-u^{2}} .
$$

Of course everything in sight is complex, much of it analytic.

For (ii), schematically the method is simply to replace in the characteristic equations, differentiation by backward differencing in the complex variables $\xi$, $\eta$. We set up a computational mesh in $\xi, \eta$ space with $\Delta \xi=\Delta \eta$, see Figure 4 .

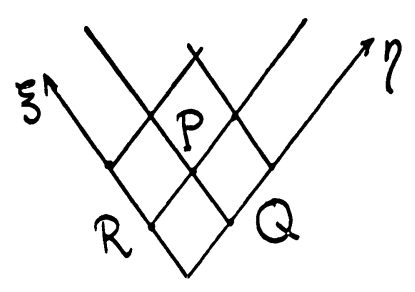

FIGURE 4. The Goursat problem

Suppose we want $(u, v)$ at $P$ (we are using the same letters for the difference scheme) in terms of values at $P, Q$. Then,

$$
\begin{aligned}
& u(P)-u(Q)-\lambda_{-}(Q)(v(P)-v(Q))=0, \\
& u(P)-u(R)-\lambda_{+}(R)(v(P)-v(R))=0 .
\end{aligned}
$$

This can be solved for $u(P), v(P)$ as long as $q^{2} \neq c^{2}$. In the complex case $q^{2}=c^{2}$ is a two-dimensional surface in four-dimensional space which we can go around, because on homologous paths our solutions are independent of path. As long as we avoid the branch points of $q^{2}-c^{2}$ everything else is holomorphic. 
Exact analogues of these solutions give us $x(P), y(P)$ in terms of their values at $Q, R$.

Of course this is a general method for continuing analytic first order systems numerically. It could be applied to a number of other inverse problems. In fluid dynamics it was used first for detached shocks corresponding to $M_{\infty}>1$ and currently it is used for other transonic problems, see [2] and bibliography therein.

We have a degree of arbitrariness in choosing the characteristic variables and it turns out that even in the elliptic region we need only three real variables instead of four. Also the numerical method is a stable marching scheme. There is no iteration. That means there are really only three computational independent real variables which makes the problem manageable on many modern computers. In addition its accuracy is easily improved.

Finally in (iii) the obvious choice for data is to give it on $\eta=$ const, $\xi=$ constant, making the problem a Goursat problem. But there are fancier ways of doing this. It will have to suffice to say that one can fix $\nabla \phi_{\infty}$ and obtain all the aerodynamic features we want by arranging the rest of the data. An example of such an airfoil, the Korn airfoil at Mach 0.750, is given in Figure 5a; along with it is a plot of a normalized speed, as a function of arc length along the airfoil, on the upper (above) and lower (below) surface. Note the presence of the supersonic bubble. The first published experimental evidence of a smooth supersonic bubble is a very convincing demonstration by Koppe and Meier [25] that the special smooth solution of (1) due to Ringleb [40] can be produced experimentally. The very first supercritical airfoils tested in wind tunnels were designed using certain underlying engineering principles about the mechanisms that create shocks in supersonic flow, see Pearcey [38] and Whitcomb [44].

A quite different method of constructing supercritical airfoils is to be found in [14]. It uses the numerical methods developed for finding the flow with shocks with side conditions to eliminate them.

So we have accomplished stage 1; we have our shock free airfoil at a specific $M_{\infty}$. How dense are they? What happens at other speeds or for nearby airfoils? To answer the second question, we study the effect of small variations.

\section{Perturbation theory.}

THEOREM. The perturbation problem for the boundary value problem for $\phi$, conditions (1)-(4), is improperly posed (overdetermined) for variations in $\partial \mathscr{D}$ under the condition $\nabla \phi \subset \mathcal{C}$ in $\mathscr{D}$. (There are some restrictions on the unperturbed airfoil and flow but they are satisfied by all constructed flows.)

Note $\nabla \phi$ continuous means no shock. Why should we expect this theorem? It was Frankl [12], Busemann [5], Guderley [19] who saw the general connection to Tricomi's linear mixed equation, $y \Phi_{x x}+\Phi_{y y}=0$, and his study of the first of its correctly posed boundary value problems [43]. Tricomi's studies were completely disconnected, as far as I can tell, from the fluid dynamics problem but they initiated, by a completed example, the study of singular integral equations. We shall look at the simpler Lavrent'ev-Bitsadze case [27], 


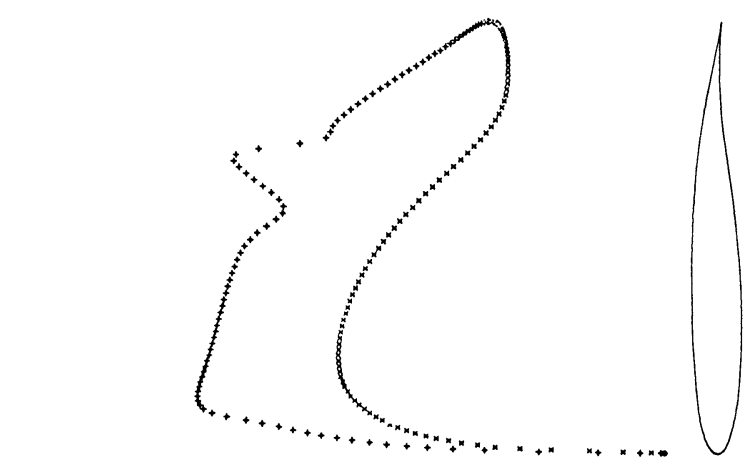

(3)

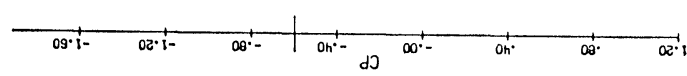

离

里

芯

है

잉

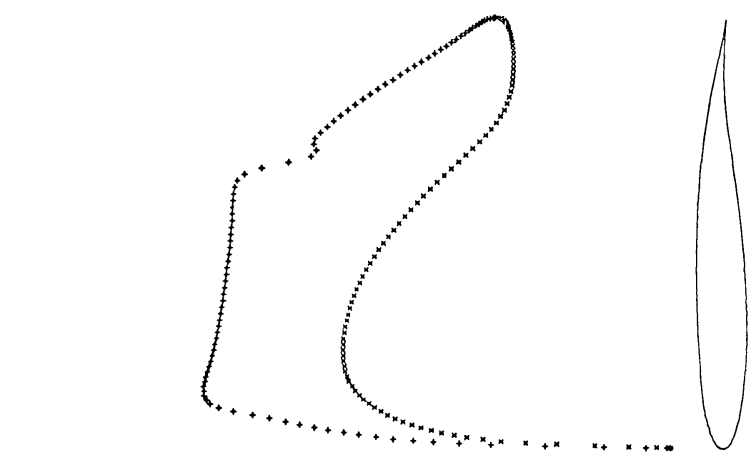

윰으

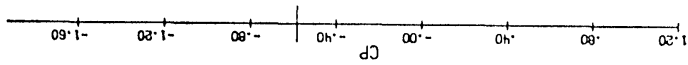

害

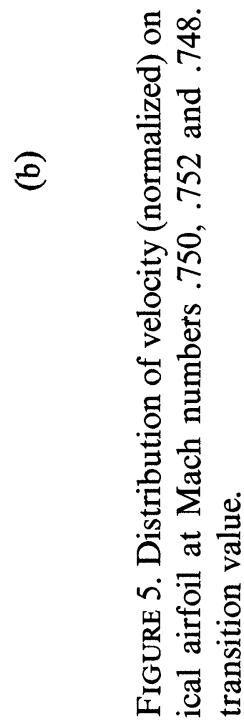

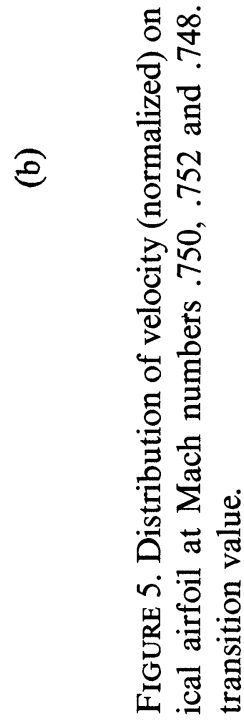

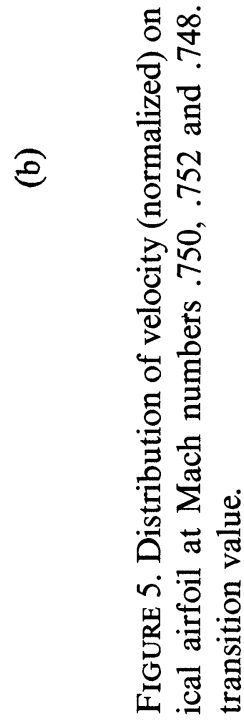

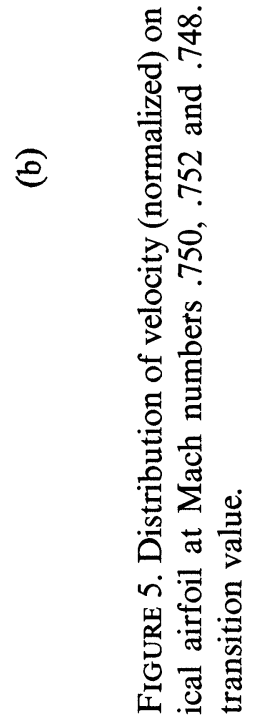
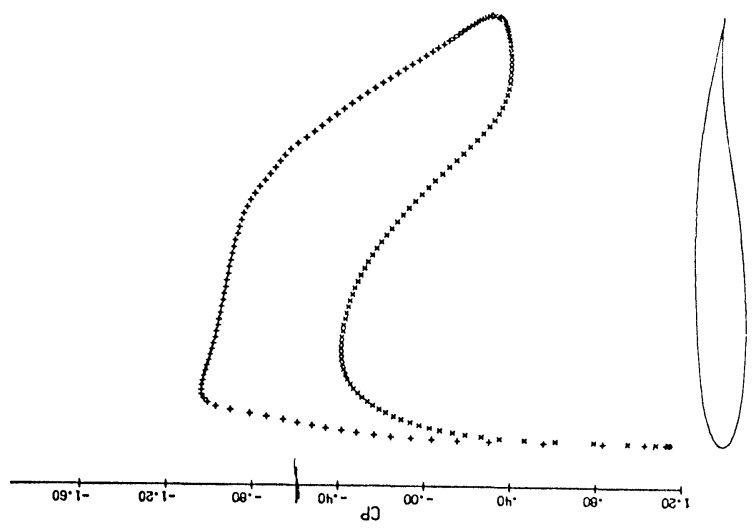

刃્త 


$$
\begin{array}{ll}
\Phi_{x x}+\Phi_{y y}=0, & y>0, \\
-\Phi_{x x}+\Phi_{y y}=0, & y<0, \\
\nabla \Phi \text { continuous across, } & y=0 .
\end{array}
$$

We shall argue ill-posedness by "counting" on a typical problem.

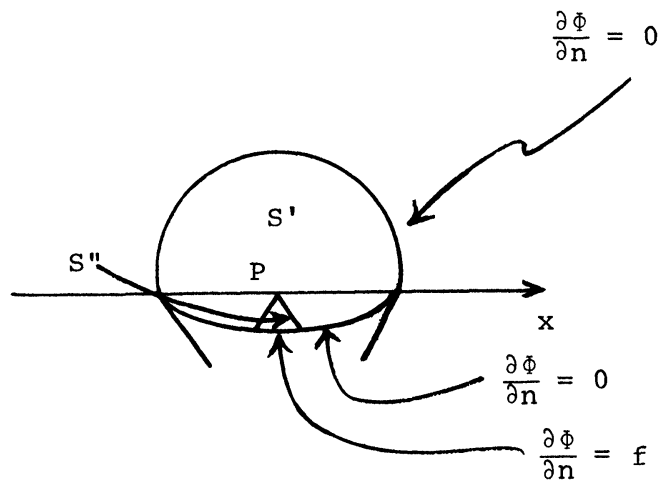

FIGURE 6. An overdetermined boundary value problem

Suppose $\Phi$ satisfies the Lavrent'ev-Bitsadze equations in a domain $S$. The domain $S$ straddles $y=0$ and is bounded by a convex curve, see Figure 6 , that is nowhere tangent to the characteristic lines $d x^{2}=d y^{2}$. We want to argue by counting that the Neumann problem is not well posed. From a point $P$, say $x=0$, on the $x$-axis inside $S$ draw the two lines, $x= \pm y$ so that $S$ is divided into $S^{\prime}$ and $S^{\prime \prime}$. On $\partial S \cap \partial S^{\prime}$ let $\partial \Phi / \partial n=0$. On $\partial S \cap \partial S^{\prime \prime}$, let $\partial \Phi / \partial n=f$. Then it is plausible that the only solutions with continuous derivatives are $\Phi \equiv$ const, $f=0$. To fix the constant take $\Phi(P)=0$. We have

(i) since $\Phi$ is harmonic in $y>0$, there exists a linear homogeneous relation between $\Phi$ on $\partial \Phi / \partial y$ on $y=0$.

(ii) Another linear relation comes from $y<0$ where $\Phi$ satisfies the wave equation and a homogeneous boundary condition on $\partial S \cap \partial S^{\prime} \cap\{y<0\}$.

(iii) From a "counting" point of view this implies $\Phi=0$ in $S^{\prime}$ and hence $\Phi=0$ on the two characteristics bounding $S^{\prime}$.

(iv) By a Goursat problem in $S^{\prime}, \Phi=0$ there.

Hence $f=0$ is the only admissible $f$.

Thus the problem appears to be ill-posed. The counting argument is slightly fallacious; there is a finite dimensional space of admissible functions $f(x)$ but that still leaves the problem ill-posed.

Now transfer this idea to our airfoil boundary value problem. Let $\delta \phi$ be the perturbation potential; as C. Gardner (unpublished) pointed out, it satisfies a linear mixed boundary value problem. Draw a pair of characteristics from the sonic line to the boundary and perturb the airfoil in the gap, Figure 7.

The counting argument works but we need a proof.

One thing simplifies the problem enormously. The equation for $\delta \phi$ is very simple in suitable variables. 


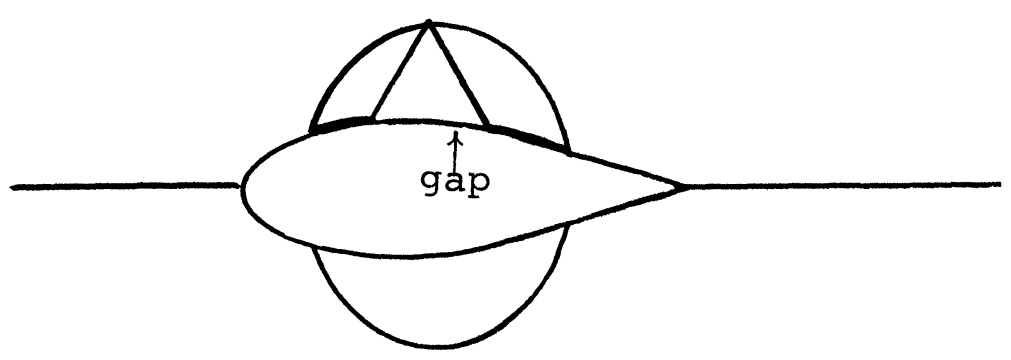

FigURE 7. The overdetermined flow problem

This comes about for an interesting general reason. Suppose $U \in R^{2}$, $X \in R^{2}$ and $U$ satisfies a nonlinear system of the form $G(U) U_{X}=0$. If we interchange variables we find because of the two-dimensionality the linear system $G(U) X_{U}=0$ for $X$. On the other hand, if $U=\nabla \phi(X)$ then there is a Legendre potential $\Phi(U)=U \cdot X-\phi(X)$ and $\nabla_{U} \Phi=X$ so that $\Phi$ satisfies a second order linear equation with given coefficients. The perturbation potential $\delta \Phi$, on the other hand satisfies $\delta \Phi+\nabla_{U} \Phi \cdot \delta U=\delta U \cdot X-\delta \phi$ or $\delta \Phi=$ $-\delta \phi$. It follows that $\delta \phi$ satisfies the same equation as $\Phi$ in the original $U$ variables.

For the perturbation problem that we have at hand this equation can be brought to a form that is remarkably like Tricomi's equation. This is very helpful in proving the perturbation theorem and in other circumstances gives one a firm hold for estimating the linearization of a nonlinear problem.

5. Linear methods. To actually prove the ill-posedness one needs somehow an estimate. One basic approach is based on Friedrichs' multiplier method, see [13] and [32].

I digress here to describe a little this method which gives the strongest known results on existence and uniqueness for linear equations of mixed type.

Suppose $U$ satisfies a first order system of partial differential equations

$$
L U=f, \quad x \in \mathscr{D},
$$

with the boundary condition $B U=0, x \in \partial \mathscr{D}$. Suppose there exists a matrix $P$ such that for smooth $V, B V=0$ the scalar product $(L V, P V)=Q(V, V)$ where $Q$ is a definite quadratic form. We can obtain (i) uniqueness for smooth solutions, (ii) an existence theorem by the projection theorem for the "adjoint" problem in some Hilbert space.

Friedrichs' approach to mixed problems was: forget about type. Does the problem at hand have such an identity leading to existence and uniqueness, or, as it was later named, is it accretive? So a new way of looking at first order linear systems was born. We get away from the standard categories. One still has to work up the smoothness to get full well-posedness and finding $P$ may be very difficult.

Let us return to our perturbation theorem for the flow. We have smoothness. We want a uniqueness theorem for the region cut out by the characteristics and the gap in Figure 7. But it turns out that we cannot find the matrix $P$. It 
suffices however to restrict the perturbations and only prove that the null-space is finite dimensional. This is all we need for ill-posedness. This is essentially my proof [33] for symmetric airfoils. The proof for nonsymmetric airfoils was given by Cooke [9]. This completes stage 2 .

6. General problem and numerical solutions. We have our airfoil, good at one Mach number; we know it produces shocks in general. ${ }^{3}$ We would like to formulate the general boundary value problem admitting shocks.

As many know, shocks mean entropy change, loss of irrotationality and the failure of the simple form of Bernoulli's law. But all these changes are of third order in the shock strength. So we keep our potential $\phi$, and the Bernoulli law and look for a weak solution satisfying, since otherwise we cannot expect uniqueness, an "entropy" condition:

$$
\text { shocks are compressive. }
$$

This natural entropy condition comes from the original gas dynamic shock conditions.

CONJECTURE. $\exists ! \phi$ satisfying the boundary value problem (1-4) and the entropy condition (5) weakly and with first derivatives of bounded variation on every streamline.

There is no proof but, as usual, the problem of real interest to engineers is to solve the boundary value problem numerically (stage 3 ).

The neighborhoods of most points in the field of flow will have either subsonic or supersonic flow and for local properties one can appeal to either elliptic or hyperbolic theory. The parabolic points are special but as a preliminary, it is helpful to know that there exist special solutions that describe the flow just at the point where the shock with vanishing strength reaches the sonic line, see P. Germain [16].

There are currently several numerical approaches to shock problems but the only one applied successfully so far to the transonic wing is the method of artificial viscosity. A variant is the penalty method used by Glowinski [18], see also Ballhaus [1], Fix [11]. It is only possible here to give the briefest idea of the method and to display some of A. Jameson's computations. Let us consider the mixed nonlinear equation $\left(\left(\operatorname{sign} \phi_{x}\right) \phi_{x}\right)_{x}+\phi_{y y}=0$ with the entropy condition: $\phi_{x}$ increases with $\phi$ at a jump. Then for the elliptic region $\Delta \phi=0$, one naturally uses centered difference operators to replace differential operators. If the equation is everywhere elliptic one then inverts for the approximate values by an iterative or relaxation method. So for the Laplacian one gets a five point scheme illustrated by the stencil

and the result is accurate to second order in the mesh size.

\footnotetext{
${ }^{3}$ The perturbation problem corresponding to changing $M_{\infty}$ has not been proved. Quite likely there are exceptional airfoils which are even more exceptional because this perturbation problem is also well-posed.
} 
But that does not quite work in the hyperbolic case and it will not pick out the solution satisfying the entropy condition in the nonlinear case. But suppose we use a stencil like this

in the hyperbolic region then we are only first order accurate in $\phi_{x x}$ but second order accurate for the operator

$$
-\phi_{x x}-\Delta x \phi_{x x x}+\phi_{y y}
$$

We have added an artificial viscosity which singles out the correct solution with some loss of accuracy. This was the very original idea of the Murman and Cole computation [35] for a similar model equation.

So we might try for $\operatorname{div}(\rho \nabla \phi)=0$ adding an artificial viscosity term, that is, develop a second order scheme to solve $\operatorname{div}(\rho \nabla \phi)+\left(\varepsilon \rho \phi_{x}\right)_{x x}=0$ with $\varepsilon=0$ where the equation is elliptic and $\varepsilon>0$ where the equation is hyperbolic. This does not quite work exactly like that except in the case studied in ColeMurman. To check the relation of $\varepsilon$ to the entropy condition, let us be still simpler and look at a one-dimensional model

$$
\left(\left(\operatorname{sign} \phi_{x}\right) \phi_{x}\right)_{x}=0 \text { with } \phi_{x}=U_{\infty}>0 \text { at }-\infty .
$$

The solution is $\phi_{x}=U_{\infty}$ or

$$
\phi_{x}= \begin{cases}U_{\infty}, & x<a, \\ -U_{\infty}, & x>a .\end{cases}
$$

Let us model the solution with $u=\phi_{x}$ and $u-(\varepsilon u)_{x}=k$ where $\varepsilon \geqslant 0$ for $u>0, \varepsilon=0$ for $u<0$. Then we get either

$$
u=U_{\infty} \text { or } u=U_{\infty}+A \exp \left\{\left(x-x_{0}\right) / \varepsilon\right\} .
$$

This solution holds until $u=0$ and then $u$ jumps to $-U_{\infty}$. Take $A=-U_{\infty}$ so that the shock is at $x_{0}$.

Thus for $\varepsilon>0$ the equation has a solution with a shock at an arbitrary point automatically satisfying the entropy condition. There is also the solution $\phi_{x} \equiv U_{\infty}$. If $U_{\infty}<0$ that is the only bounded solution that satisfies the entropy condition. Hence in the first case we can throw in another condition on $\phi$. That appears to be the freedom we need in the two-dimensional problem.

Adding regularizing or viscous terms to the equation is used in practice for hyperbolic problems. Oleinik [37] and Kruskov [20] have shown that in the scalar first order case, and in some second order strictly hyperbolic cases, initial value problems can be solved by the viscosity method. But our problem has not lent itself to proof this way so far.

Let us proceed to the numerical results:

Figure 8 shows a Joukowski airfoil with two symmetric shocks, quite strong. Strong enough to see in the figure but not strong enough to really contradict our assumptions. 


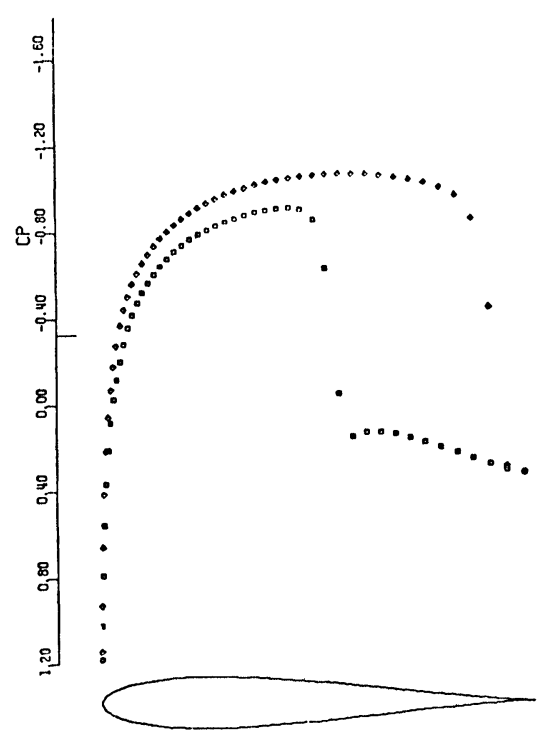

FIGURE 8. Joukowski airfoil

Now we look at the good airfoil of Figure 5 again. The graph of the pressure distribution here is actually not computed by the design but by the Jameson code. But it is exactly the same to the eye.

Next we shall check our theorem on ill-posedness; we just change the Mach number ${ }^{4}$ a little bit. We put it up $0.3 \%$ and obtain the distinct shock of Figure $5 \mathrm{~b}$. We put it down the same amount and there is definitely again a shock but also much more distortion (Figure 5c). The computational results give very sharp shocks which match the wind tunnel results. There is much work on improving the difference schemes and on the iteration scheme used in inverting the matrix, see the work of Mock [31], and Engquist and Osher [10] who show this iteration converges.

Notice, from Figure 5b, however, that there is very little change in the flow elsewhere if we raise the Mach number. This appears to be so in general. There ought to be a linear perturbation potential that holds away from the sonic boundary point $P$.

So for increases in Mach number there should exist a perturbation potential with a singular point at $P$. There are in fact some model problems [34] of this kind (push the "gap" in Figure 6 over to the boundary). We could speculate even further. There ought to be an inner and outer expansion for the perturbation potential. The inner should be a shock at Mach 1 .

But we must keep in mind that in general the computed flow patterns may give rise to many small shocks, even to shocks entirely embedded in the supersonic region, see Garabedian and McFadden [15].

\footnotetext{
${ }^{4}$ These figures are obtained by using a very fine mesh.
} 
This lecture began with the drag and now is the time to see what the computed drag and lift are. In incompressible flow one uses contour integrals to obtain the elegant formulas of Blasius for lift and drag in terms of the second coefficient in the power series that describes the complex velocity at $\infty$. (The famous paradox of d'Alembert is that the drag is zero because there is no viscosity.) In our case, we can obtain the force on the wing as two line integrals - one along the shock, the other "at $\infty$ " by a clever integration by parts. The drag on a symmetric airfoil is given in this way in terms of the loss of momentum in the normal direction to the shock. This would have been conserved if we had not thrown away that third order change in entropy. So the drag is of third order in the shock strength. That makes it small which is good but it also means that at this stage we must rely on the wind tunnel to measure the accuracy with which we are getting the drag. For this purpose a variety of numerical schemes, in conservation (i.e. divergence) form are available to choose from. Remarkably, the measured drag is very close to the computed drag but it would be better if there were a mathematical justification.

7. Conclusion. We are left with the general weak existence theorem for the full nonlinear problem unsolved. There are lots of approaches to try: show the difference scheme converges. Extend the variational principles of elliptic theory. Perhaps something quite new.

Numerically there have been many advances. There are now threedimensional computations and time dependent flow. Perhaps a whole airplane and its flow will some day be computed-the ultimate answer to the wind tunnel and much cheaper. From all these numerical experiments we are gaining understanding of all the possible phenomena. Perhaps that too will help with the theorems.

Let me close now with two things. The first is to say that I have left a lot unsaid and a lot unquoted. But I would like to thank for their help my transonic colleagues, Kurt O. Friedrichs, Lipman Bers, Paul R. Garabedian and Antony Jameson.

Finally, a story about Theodore v. Karman, who gave much to early transonic theory, see [23]. In his Gibbs Lecture of 1940 [24], he proposed, as one of many nonlinear problems of importance to engineers, the resolution of the paradox of wing theory that I have been describing. The story is told that someone once asked von Karman where he got his ideas. He replied, "physical intuition, physical intuition" patting at the same time, a very large pile of mathematical calculations. I think today he would have in that pile a very large amount of computer output.

ACKNOWLEDGMENTS. Figure 1 is from Jones' book, [22]. The photographs in Figure 2 were made in a N.A.S.A. wind tunnel at Langley Field; (a), (b), (d) are of a supercritical wing section designed by Bauer and Korn using the method of Garabedian [2]. Figure $2 \mathrm{c}$ is of a wing section by Bauer and Korn that imitates a supercritical wing design of Whitcomb [44]. Figures 5 and 8 were made by A. Jameson with his code. 


\section{BIBLIOGRAPHY}

Introductions to transonic gas dynamics and the related problems for partial differential equations of mixed type can be found in the following books, all of which have very complete bibliographies.

L. Bers, Mathematical aspects of subsonic and transonic gas dynamics, Wiley, New York, 1958.

A. V. Bitsadze, Equations of the mixed type, Translated by P. Zador, MacMillan, New York, 1964.

G. Guderley, Theorie Schallnaher Störmungen, Springer-Verlag, 1957. Theory of transonic flow, Pergamon Press, New York, 1952.

A. R. Manwell, The Hodograph equations, Hafner Publishing, New York, 1971.

More recent review articles in the subject are:

J. Cole, Modern developments in transonic flow, SIAM J. Appl. Math. (4) 29 (1975), 763-787.

C. S. Morawetz, Mixed equations and transonic flow, Rend. Mat. e Appl. (5) 25 (1966), 482-509. Well-posed problems and transonic flow, Fluid Dynamics Transactions, Polish Academy of Sciences, 6, Part I, 1972, pp. 325-333.

Internat. Union of Theoretical and Applied Mechanics, Symposium Transsonicum, SpringerVerlag, Berlin and New York, Aachen, 1962, K. Oswatitsch (ed.), 1964, Göttingen, 1975, K. Oswatitsch and D. Rues (eds.), 1976.

Articles on the state of computational transonic flow are to be found in "Numerical Methods in Fluid Dynamics", Hemisphere Publishing Corporation, 1978, H. J. Wirz and J. J. Smolderen (eds.)

W. F. Ballhaus, Some recent progress in transonic flow computations, pp. 155-236.

W. Schmidt, Progress in transonic flow computations. Analysis and design methods for three dimensional flows, pp. 299-335.

A. Jameson, Transonic flow computations, pp. 1-154.

For the relevant background on gas dynamics in a mathematical setting the reader is referred to:

R. Courant and K. O. Friedrichs, Supersonic flow and shock waves, Interscience, New York, 1948.

1. W. F. Ballhaus and P. M. Goorian, Implicit finite difference computations of unsteady transonic flows about airfoils, A.I.A.A. Journal 15 (1977); see also 17 (1979).

2. F. Bauer, P. Garabedian and D. Korn, A theory of supercritical wing sections with computer programs and examples, Lecture Notes in Econom. and Math. Syst., vol. 66, Springer-Verlag, Berlin and New York, 1972. See also with A. Jameson, II, 108, same series and III, 150, same series.

3. L. Bers, Existence and uniqueness of a subsonic flow past a given profile, Comm. Pure Appl. Math. 7 (1945), 441-504.

4. H. Brezis and G. Stampacchia, The hodograph method in fluid dynamics in the light of variational inequalities, Arch. Rational Mech. Anal. 61 (1976), 1-18.

5. A. Busemann, Widerstand bei geschwindigkeiten naher der schallgeschwindigkeiten, Proc. Third Internat. Congr. Appl. Mech. 1 (1930), 282-285.

6. __ The nonexistence of transonic potential flow, Proc. Sympos. Appl. Math., vol. 4, 1953, pp. $282-285$.

7. S. A. Chaplygin, On gas jets, Sci. Mem. Moscow Univ. Math. Phys., No. 21, 1904, pp. 1-21. Trans. NACA TM. 1063 (1944).

8. T. M. Cherry, Flow of a compressible fluid about a cylinder, Proc. Roy. Soc. London Ser. A 192 (1947), 45-79; A 196 (1949), 1-31; 32-36.

9. Pamela Cooke, $A$ uniqueness proof for a transonic flow problem, Indiana Univ. Math. J. 27 (1978), 51-71.

10. R. Engquist and S. Osher, Stable and entropy satisfying approximations for transonic flow calculations, Math. Comp. 34 (1980), 45-75.

11. G. J. Fix, A mixed finite element scheme for transonic flows, ICASE, Hampton, Virginia, Report 76-25, 1976. 
12. F. I. Frankl, On the appearance of compression shocks in subsonic speeds, Prikl. Mat. Meh. 11 (1947), 199-202.

13. K. O. Friedrichs, Symmetric positive linear differential equations, Comm. Pure Appl. Math. 11 (1958), 333-418.

14. K. Y. Fung, H. Sobieczky and R. Seebass, Shockfree wing design, A.I.A.A. Journal 18, Article No. 79-1557R, 1980.

15. P. Garabedian and G. McFadden, Design of supercritical swept wings (preprint).

16. P. Germain, Ecoulements transsoniques homogênes, Progress in Aeronautical Sciences, D. Küchenbaum and L. H. G. Sterne (eds.), Pergamon Press, New York, 1964.

17. D. Gilbarg, Comparison methods in the theory of subsonic flows, J. Rational Mech. Anal. 2 (1953), 223-251.

18. R. Glowinski and O. Pironneaux, Calcul d'écoulements transonics par des méthodes d'elements finis et de contrôle optimal, Computing Methods in Applied Sciences and Engineering (Sec. Internat. Sympos., Versailles), Part I, Lecture Notes in Econom. and Math. Systems, no. 134, Springer-Verlag, New York, 1976.

19. G. Guderley, On the presence of shocks in mixed subsonic-supersonic flow patterns, Adv. in Appl. Mech. 3 (1953), 145-184.

20. R. A. Hummel, The hodograph method for convex profiles (to appear).

21. A. Jameson, Iterative solution of transonic flow over airfoils and wings including flows at Mach

1, Comm. Pure Appl. Máth. 27 (1974), 283-309.

22. R. T. Jones, High speed wing theory, Princeton Univ. Press, Princeton, N.J., 1960.

23. T. von Karman, Compressibility effects in aerodynamics, J. Aeronaut. Sci. 8 (1941), 337-356. 24. , The engineer grapples with nonlinear problems, Bull. Amer. Math. Soc. 46 (1940), 615-683.

25. E. Koppe and G. Meier, Erfahrungen mit optischen methoden bei der Untersuchung transonischer Strömungen, Jahrgang 5 (1965), 150-157.

26. S. N. Kruzkov, First order quasilinear equations in several independent variables, Mat. Sb. (N.S.) 81(123) (1970), 228-255, Amer. Math. Soc. Transl. (1971).

27. M. A. Lavrent'ev and A. Bitsadze, On the problem of equations of mixed type, Doklady 70 (1950), 373-376. See also pp. 561-564.

28. M. J. Lighthill, On the hodograph transformation for high-speed flow. II. A flow with circulation. Quart. J. Mech. Appl. Math. 1 (1948), 442-450. The hodograph transformation in trans-sonic flow. III. Flow around a body, Proc. Roy. Soc. London Ser. A 191 (1947), 341-369.

29. R. Magnus and H. Yoshihara, Inviscid transonic flow over airfoils, A.I.A.A. Journal 8 (1970), 2157-2162.

30. Th. Meyer, Über zwei dimensionale Bewegungvorgange in einem Gas das mit Überschallgeschwingigkeitstrom, Forschungs hefte 62 (1908).

31. M. S. Mock, Systems of conservation laws of mixed type, J. Differential Equations 37 (1980), $70-88$.

32. C. S. Morawetz, A weak solution for a system of equations of elliptic-hyperbolic type, Comm. Pure Appl. Math. 11 (1958), 315-332.

33. On the non-existence of continuous transonic flows past profiles. I, Comm. Pure Appl. Math. 9 (1956), 45-68; II 10 (1957), 107-132; III 11 (1958), 129-144. See also 17 (1964), 357-367.

34. The Dirichlet problem for the Tricomi equation, Comm. Pure Appl. Math. 23 (1970), 587-601.

35. E. M. Murman and J. D. Cole, Calculation of plane steady transonic flows, A.I.A.A. Journal 9 (1971), 114-121.

36. G. Y. Nieuwland, The computation by Lighthill's method of transonic potential flow around a family of quasi-elliptical aerofoils, Netherland N.L.R. Tech. Rep. T83, (1964). See also T.172.

37. O. A. Oleinik, Quasi-linear second order parabolic equations with many independent variables, Seminari 1962-63 di Analisi, algebra, geometria e topologia. Istituto Nazionale di alta matematica Edizione Cremonese, 1965.

38. H. H. Pearcey, The aerodynamic design of section shapes for swept wings, Adv. in Aeronautical Sciences 3 (1962) 277-320.

39. J. Rayleigh, On the flow of a compressible fluid past an obstacle, Philos. Mag. 32 (1916), 1-6. 
40. F. Ringleb, Exakte Lösungen der Differential gleichungen einer adiabatischen Gasströmung, Zeitschrift für angewandte Mathematik und Mechanik 20 (1940), 185-198.

41. M. Shiffman, On the existence of subsonic flows of a compressible fluid, J. Rational Mech. Anal. 1 (1952), 605-652.

42. G. I. Taylor, The flow around a body moving in a compressible fluid, Proc. Third Internat. Congr. Appl. Mech. 1 (1930), 263-275.

43. F. Tricomi, Sulla equazione lineari alle derivate parziali di secondo ordine, di tipo misto, Rendiconti Atti del Accademia Nazionale dei Lincei, Series 514 (1923), 134-247.

44. R. T. Whitcomb, Review of NASA supercritical airfoils, Ninth Internat. Congr. Aeronautical Sciences, Haifa, 1974.

Courant Institute of Mathematical Sciences, New York University, New York 10012 
